\title{
ARGILAS COMO CATALISADORES VERDES NA ESTERIFICAÇÃO DO COLESTEROL. CARACTERIZAÇÃO ESPECTROSCÓPICA E IDENTIFICAÇÃO DE POLIMORFOS POR MÉTODOS DE ANÁLISE TÉRMICA. UMA PROPOSTA LABORATORIAL INTERDISCIPLINAR PARA O $1^{\circ}$ CICLO UNIVERSITÁRIO
}

\author{
Teresa M. R. Maria*, Rui M. D. Nunes, Mariette M. Pereira e M. Ermelinda S. Eusébio \\ Departamento de Química, Universidade de Coimbra, 3004-535 Coimbra - Portugal
}

Recebido em 6/1/09; aceito em 7/4/09; publicado na web em 22/9/09

\begin{abstract}
CLAYS AS GREEN CATALYSTS IN THE CHOLESTEROL ESTERIFICATION. SPECTROSCOPIC CHARACTERIZATION AND POLYMORPHS IDENTIFICATION BY THERMAL ANALYSIS METHODS. AN INTERDISCIPLINARY LABORATORIAL PROPOSAL FOR THE UNDERGRADUATE LEVEL. A laboratory experiment that enables the professor to introduce the problematic of sustainable development in pharmaceutical chemistry to undergraduate students is proposed, using a simple synthetic procedure. Cholesteryl acetate is prepared by the esterification of cholesterol using Montmorillonite K10 as heterogeneous catalyst. Cholesterol and cholesteryl acetate are characterized by spectroscopic $\left({ }^{1} \mathrm{H}\right.$ RMN, ${ }^{13} \mathrm{C}$ RMN, FTIR) and thermal analysis techniques. The thermal methods are used to introduce the concepts of polymorphism and the nature of mesophases.
\end{abstract}

Keywords: green chemistry; cholesteryl acetate; polymorphism.

\section{INTRODUÇÃO}

Os esteroides são uma família de compostos naturais com múltiplas funções biológicas, nomeadamente na síntese de hormonas sexuais e adreno-corticais, glicocorticoides cardíacos e ainda ácidos biliares. O colesterol é um intermediário na biogénese dos diversos esteróides e, tal como os terpenos, é constituído bloco a bloco a partir de unidades de isopreno.

O colesterol é um dos esteróides mais abundantes nos tecidos humanos, especialmente no cérebro e espinal-medula. Tendo em conta as múltiplas funções das moléculas de esteroides, está sempre presente o desafio de desenvolver novas estratégias que conduzam à sua derivatização, para assim modular as respectivas propriedades biológicas. De entre as possíveis derivatizações, os ésteres sintéticos de moléculas de esteroides são compostos com interesse crescente atendendo às suas relevantes aplicações a nível farmacológico, descobertas nas últimas décadas. ${ }^{1}$

Actualmente é de consenso global a necessidade de desenvolver processos químicos ambientalmente sustentáveis, tal como acordado por 192 países no Rio de Janeiro em 1992 (ECO 92) e por 172 países no protocolo de Quioto em 1997. Neste contexto, ao químico sintético impõe-se o desafio de promover o desenvolvimento de novos métodos de síntese, mais eficientes e menos poluentes. Para este fim, o recurso a catalisadores reutilizáveis mereceu um acrescido interesse por parte de académicos e industriais. ${ }^{2}$

Trost introduziu o conceito de economia atómica ${ }^{3}$ onde refere que numa síntese ideal a maioria dos átomos dos reagentes deve ser incorporada nos produtos finais. Efectuando uma análise destes princípios em termos de factor $\boldsymbol{E}$ ( $\boldsymbol{E}=$ massa de produtos secundários/ massa do produto desejado, em $\mathrm{kg}$ ) verifica-se que este factor aumenta consideravelmente com a especificidade da indústria, Tabela 1.

Numa perspectiva de interdisciplinaridade, o colesterol e os seus ésteres são também compostos privilegiados para a discussão de temas de interesse transversal em ciência, como são o polimorfismo e as mesofases (estados físicos da matéria com grau de ordem intermédio entre o estado sólido cristalino e o líquido isotrópico). De facto, o colesterol pode apresentar diferentes formas cristalinas, isto é, exibe

\footnotetext{
*e-mail: troseiro@ci.uc.pt
}

Tabela 1. Factor E em diferentes tipos de indústria*

\begin{tabular}{lcc}
\hline Indústria & Produção (t/ano) & Factor E \\
\hline Petrolífera & Dezenas de milhões & 0.1 \\
Química Pesada & Centenas de milhar & 1 a 5 \\
Química Fina & Milhares & 5 a 50 \\
Química Farmacêutica & Centenas & 25 a 100 \\
\hline
\end{tabular}

*Adapatado de Bolm, C.; Beckmann, O. A.; Angew Int. Ed. Engl. 1999, 38, 907

polimorfismo. ${ }^{4-7}$ A pesquisa e caracterização dos polimorfos de uma dada substância é da maior importância em diversos ramos da ciência e da indústria, sendo de realçar o seu papel na indústria farmacêutica. ${ }^{8,9}$ Propriedades tais como a solubilidade, a velocidade de dissolução, que afectam a biodisponibilidade de um fármaco, entre outras, dependem da forma polimórfica em que o composto activo do medicamento se encontra. ${ }^{8,10}$ Muitos ésteres de colesterol, para além de apresentarem diferentes formas cristalinas, têm, também, mesofases, no caso do acetato de colesterol uma mesofase cristal líquido colestérico. ${ }^{11-13}$

Trabalhos laboratoriais com a combinação de várias métodos de estudo não são comuns, embora haja exemplos. ${ }^{14}$ Neste artigo descrevemos uma experiência laboratorial que combina a síntese de um éster do colesterol utilizando um catalisador reutilizável, Montmorillonite K10, com a caracterização espectroscópica e por métodos de análise térmica do produto final e/ou reagentes de partida.

\section{ESTRATÉGIA DAS AULAS}

Esta proposta de projecto laboratorial foi desenvolvida no Departamento de Química da Universidade de Coimbra, Portugal, envolvendo alunos do $3^{\circ}$ ano das licenciaturas em Química e em Química Industrial. O projecto foi desenvolvido por um grupo de 12 alunos que foram organizados aos pares, tendo decorrido durante 4 aulas de $3 \mathrm{~h}$.

Este projecto é interdisciplinar e permite a integração dos conceitos de química sustentável com os de catálise, síntese, identificação e caracterização de compostos orgânicos por técnicas espectroscópicas e de análise térmica. 


\section{Esterificação do colesterol}

A esterificação é uma reacção bem compreendida e utilizada especialmente pelas indústrias farmacêutica, de perfumaria e fragrâncias. ${ }^{15}$ Existem vários processos de síntese de ésteres mas, um dos métodos mais utilizados, é a reacção de um álcool com um anidrido de um ácido carboxílico. Esta reacção, para ocorrer a uma velocidade operacional, requer o recurso a catalisadores. Por outro lado, para que a reacção ocorra extensamente é normal utilizar um excesso de um dos reagentes ou remover continuamente o éster formado ou um dos coprodutos. Em muitos dos trabalhos laboratoriais dos primeiros anos dos cursos de química universitária, procede-se à esterificação de álcoois recorrendo à utilização de quantidades catalíticas de ácidos minerais fortes. Estes ácidos, nomeadamente sulfúrico e clorídrico, são altamente corrosivos, podendo causar problemas de saúde aos alunos, assim como problemas ambientais.

Mais recentemente, já numa perspectiva de química verde, as argilas do tipo Montmorillonite, foram utilizadas como catalisadores ácidos, de baixo custo, não corrosivos e reutilizáveis, em reacções de acetilação de álcoois. ${ }^{16}$

No primeiro dia de aulas os 6 grupos de trabalho apresentaram oralmente, durante $10 \mathrm{~min}$, a proposta de síntese que iam realizar, assim como o material necessário. Após discussão entre o professor e todos os alunos, acerca do mecanismo da reacção de esterificação, Esquema 1, e sensibilização para questões de desenvolvimento sustentável, envolvendo catalisadores reutilizáveis, deu-se início ao trabalho de síntese. Cada grupo realizou a síntese de acordo com o procedimento apresentado detalhadamente na secção experimental. Na segunda aula, procedeu-se à recuperação do catalisador recorrendo à técnica da filtração. Após work-up e evaporação do solvente, no evaporador rotativo, procedeu-se à recristalização para obter cristais puros.

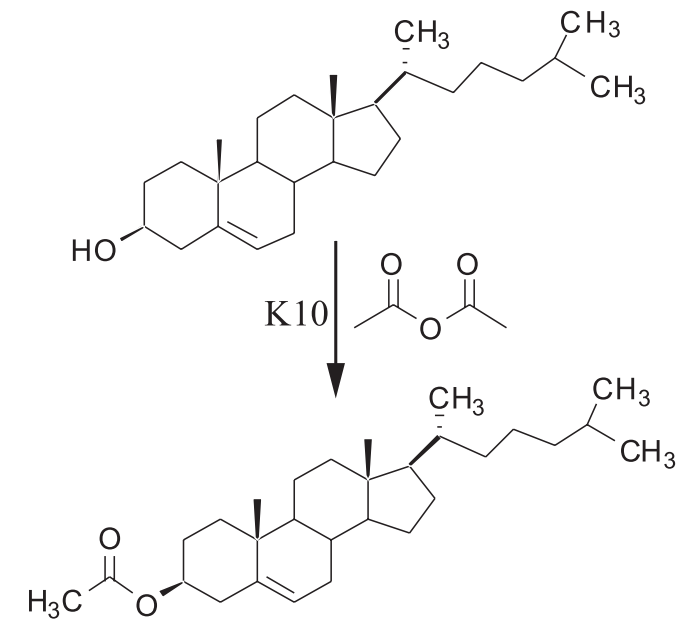

Esquema 1.

\section{Análise espectroscópica}

Na terceira aula os alunos efectuaram a caracterização recorrendo às técnicas de ressonância magnética nuclear protónica e de ${ }^{13} \mathrm{C}$ e à espectroscopia de infravermelho, seguindo-se discussão dos resultados.

\section{Espectroscopia de ressonância magnética nuclear}

Na Figura 1 apresenta-se o espectro de ${ }^{1} \mathrm{H}$ RMN do acetato de colesterol sintetizado pelos alunos. No espectro pode facilmente identificar-se o sinal a $\delta=5,35$ ppm com integração de 1 , correspondente ao protão vinílico $\mathrm{H}-6$; o sinal a $\delta=4,55$ ppm do protão $\mathrm{H}-3$, com integração de 1 , que mostra um desvio acentuado do protão
H-3 no colesterol a $\delta=3,50 \mathrm{ppm}$; o sinal a $\delta=2,03$, com integração 3 , típico do grupo metilo do éster e que não se observa no espectro do colesterol. Para além dos multipletos compreendidos entre $\delta=2,3$ e $1,0 \mathrm{ppm}$, salientamos ainda os sinais a $\delta=1,01 ; 0,90 ; 0,88 ; 0,87$ e 0,67 identificados como os grupos metilo na posição C-19, C-21, C-26 e C-18 respectivamente. ${ }^{17}$

$\mathrm{Da}$ análise deste espectro pode concluir-se que no decorrer do trabalho se obteve o éster metílico do colesterol com a estrutura pretendida.

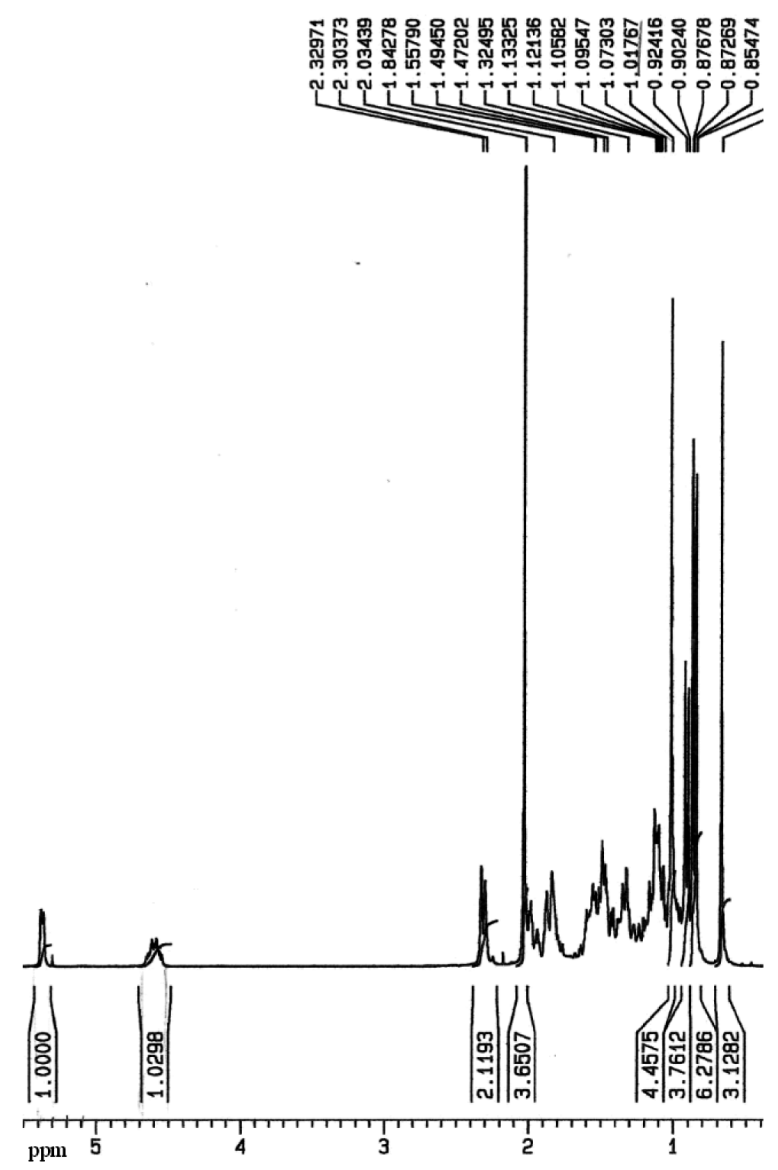

Figura 1. Espectro de ${ }^{1} \mathrm{H}$ RMN de acetato de colesterol em $\mathrm{CDCl}_{3}$

No espectro de RMN de ${ }^{13} \mathrm{C}$ do acetato de colesterol pode identificar-se a presença de um grupo carbonilo, a $\delta=170,5 \mathrm{ppm}$, que não existe no espectro de ${ }^{13} \mathrm{C}$ do colesterol; o carbono quaternário $\mathrm{C}-5$, a $\delta=139,6$ ppm, ligeiramente desviado em comparação ao do colesterol a $\delta=142,0$ ppm; o carbono vinílico C- 6 a $\delta=122,6$ ppm; o carbono C-3 aparece a $\delta=73,9 \mathrm{ppm}$, desviado 2,3 ppm em relação ao colesterol.

\section{Espectroscopia de infravermelho}

$\mathrm{Na}$ Figura 2 estão representados os espectros de infravermelho do colesterol e do acetato de colesterol, ambos obtidos em pastilha de brometo de potássio. A distinção dos dois compostos é facilmente conseguida já que os espectros são significativamente diferentes. $\mathrm{O}$ colesterol apresenta uma banda larga entre 3250 e $3500 \mathrm{~cm}^{-1}$ devida à vibração de elongação da ligação O-H. Este pico não se encontra presente no espectro do acetato de colesterol uma vez que este não tem nenhum grupo hidroxilo. A região abaixo de $2000 \mathrm{~cm}^{-1}$ também apresenta diferenças. De notar, no espectro do acetato de colesterol as bandas intensas situadas a 1750 e $1250 \mathrm{~cm}^{-1}$, correspondentes à vibração de elongação das ligações $\mathrm{C}=\mathrm{O}$ e $\mathrm{C}-\mathrm{O}$, respectivamente, as quais não são observadas no espectro do colesterol. 


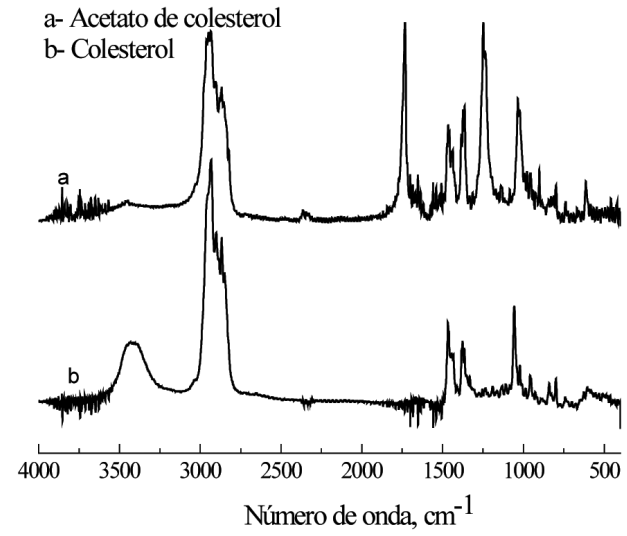

Figura 2. Espectros de infravermelho de (a) acetato de colesterol e (b) colesterol, obtidos em pastilha de $\mathrm{KBr}$

\section{Análise térmica}

Na quarta aula, são efectuados os estudos por calorimetria diferencial de varrimento e termomicroscopia com luz polarizada e a respectiva discussão.

Os métodos de análise térmica têm um papel privilegiado no estudo do polimorfismo. Dentre eles, a calorimetria diferencial de varrimento é de extrema utilidade já que, é possível recolher informações sobre modificações estruturais no decorrer dos processos de aquecimento e arrefecimento dos materiais em estudo. As transições de fase, envolvendo o sólido, põem em evidência a estrutura através das propriedades termodinâmicas que caracterizam tais transformações (temperatura de transição, variação de entalpia ou de capacidade calorífica).

A termomicroscopia é um método que permite o acompanhamento das alterações produzidas numa amostra por variação de temperatura através de observação microscópica com luz polarizada. Este método permite recolher grande número de informações sob o ponto de vista estrutural, tais como distinção entre meios anisotrópicos e isotrópicos, hábitos cristalinos e tipos de transformação de fase. É pois, de grande utilidade, e um método complementar do DSC.

\section{Colesterol}

As curvas de DSC obtidas nos ensaios de aquecimento/arrefecimento de uma amostra de colesterol, cristalizado em diclorometano/metanol (1:2), estão ilustradas na Figura 3. No processo de aquecimento observam-se duas transformações endotérmicas. A transformação registada a $39,7{ }^{\circ} \mathrm{C}$ corresponde a uma transição sólido-sólido na qual ocorre a conversão de uma forma cristalina de colesterol numa outra. O segundo pico, registrado a $144,3{ }^{\circ} \mathrm{C}$ corresponde à fusão do segundo polimorfo do colesterol. O processo de fusão é acompanhado por uma variação de entalpia igual a $25,3 \mathrm{~kJ} \mathrm{~mol}^{-1}$. A entropia de fusão é igual a $60,6 \mathrm{~J} \mathrm{~mol}^{-1} \mathrm{~K}^{-1}$ típico da fusão de cristais orgânicos. ${ }^{18,19}$ No processo de arrefecimento registra-se um pico exotérmico, a $115,2{ }^{\circ} \mathrm{C}$, o qual corresponde à cristalização do colesterol na forma polimórfica que funde a temperatura mais elevada. No processo de arrefecimento não foi observada a transformação sólido-sólido uma vez que o arrefecimento terminou a $25^{\circ} \mathrm{C}$, e esta transição apresenta um elevado sobrearrefecimento..$^{20}$

As imagens apresentadas na Figura 4 ilustram o comportamento do composto no processo de aquecimento. A transformação sólidosólido é evidente nas imagens (a) onde se observam diferentes texturas e coloração resultantes da alteração da estrutura cristalina do composto. As imagens (b) ilustram o processo de fusão.

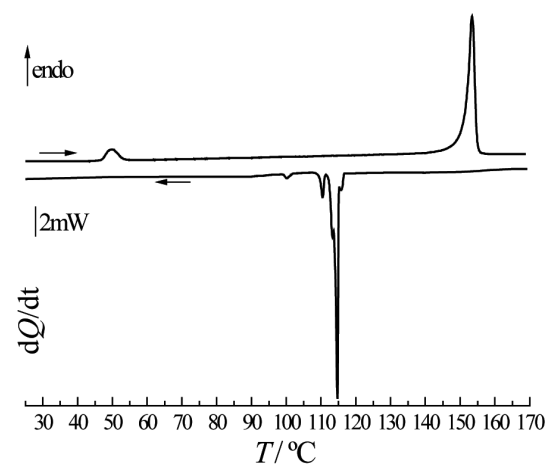

Figura 3. Curvas de DSC de aquecimento/arrefecimento de colesterol. $m=$ $2,47 \mathrm{mg}$. Velocidade de aquecimento $10^{\circ} \mathrm{C} \mathrm{min}^{-1}$ (a)

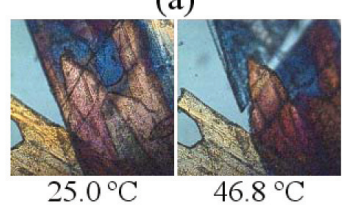

(b)

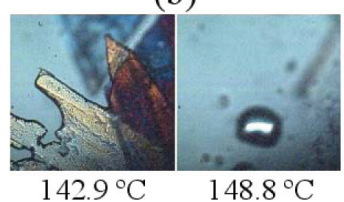

Figura 4. Imagens obtidas por termomicroscopia com luz polarizada durante o processo de aquecimento de uma amostra de colesterol. (a) Transição sólido/ sólido; (b) fusão. Velocidade de aquecimento $10^{\circ} \mathrm{C}$ min $^{-1}$. Ampliação $200 x$

\section{Acetato de colesterol}

$\mathrm{O}$ acetato de colesterol pode apresentar várias formas cristalinas e também uma mesofase descrita como cristal líquido colestérico. ${ }^{11-13}$ O termo colestérico deriva do facto de este tipo de fase ter sido identificada pela primeira vez no benzoato de colesterol. ${ }^{21}$ Esta mesofase é observada em compostos quirais nos quais as moléculas permanecem paralelas umas às outras num dado plano, mas entre planos há uma pequena rotação do arranjo molecular relativamente ao plano precedente donde resulta uma estrutura helicoidal.

Na Figura 5 estão ilustradas as curvas de DSC obtidas num processo de aquecimento/arrefecimento do acetato de colesterol cristalizado numa mistura diclorometano/acetato de etilo (1:10) (agulhas). Neste ensaio o sólido foi aquecido até $120^{\circ} \mathrm{C}$ e arrefecido de imediato até à temperatura ambiente $\left(10^{\circ} \mathrm{C} / \mathrm{min}\right)$. No processo de aquecimento são observadas duas transformações endotérmicas. O pico a temperatura mais baixa, $T_{\text {trs }}=75,7^{\circ} \mathrm{C}, \Delta_{\text {trs }} H=5 \mathrm{~kJ} \mathrm{~mol}^{-1}$, diz respeito à transformação entre fases sólidas cristalinas. $\mathrm{O}$ pico registado a temperatura mais alta, $T_{\text {fus }}=107,1^{\circ} \mathrm{C}, \Delta_{\text {fus }} H=17 \mathrm{~kJ} \mathrm{~mol}^{-1}$, corresponde à fusão da forma cristalina estável a temperatura mais elevada. No processo de arrefecimento a partir do fundido observa-se um pico exotérmico, $T_{\mathrm{cr}}=98,2^{\circ} \mathrm{Ce} \Delta_{\mathrm{cr}} H=15 \mathrm{~kJ} \mathrm{~mol}^{-1}$, o qual corresponde à transformação do líquido numa fase sólida cristalina. $\mathrm{O}$ arrefecimento até $25^{\circ} \mathrm{C}$ não revelou qualquer outra transição de fase. $\mathrm{O}$ reaquecimento do material obtido deu origem à curva de DSC (b) apresentada na Figura 5 registando-se apenas uma única transição endotérmica, $T_{\text {fus }}=106,5$ ${ }^{\circ} \mathrm{C}$ e $\Delta_{\text {fus }} H=16,7 \mathrm{~kJ} \mathrm{~mol}^{-1}$.

As imagens apresentadas na Figura 6 ilustram o comportamento registrado no aquecimento.

No processo de arrefecimento de amostras aquecidas até $120^{\circ} \mathrm{C}$, registraram-se por termomicroscopia dois tipos de comportamento: o líquido cristaliza numa única forma originando um sólido que funde a $\mathrm{T} \approx 107^{\circ} \mathrm{C}$, ou sob duas formas cristalinas diferentes, com temperaturas de fusão $\mathrm{T} \approx 92{ }^{\circ} \mathrm{C}$ e $\mathrm{T} \approx 106^{\circ} \mathrm{C}$.

Em compostos que exibem polimorfismo as fases sólidas obtidas dependem da história térmica da amostra. No caso do acetato de colesterol a mesofase cristal líquido colestérico pode ser obtida aquecendo 
o fundido a $160{ }^{\circ} \mathrm{C}$ e realizando o arrefecimento subsequente a uma velocidade relativamente baixa $\left(2,5^{\circ} \mathrm{C} / \mathrm{min}\right) .{ }^{13} \mathrm{O}$ comportamento registado no arrefecimento, nestas condições, encontra-se ilustrado na Figura 7. Ao atingir-se a temperatura de aproximadamente 73,1 ${ }^{\circ} \mathrm{C}$ tem início a formação da fase cristal líquido (a), a qual a cerca de $67,6{ }^{\circ} \mathrm{C}$ se transforma numa fase sólida cristalina (b).

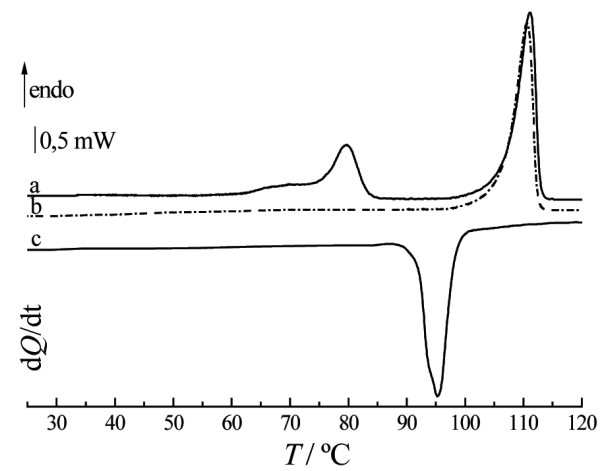

Figura 5. Curvas de DSC de aquecimento/arrefecimento de acetato de colesterol. (a) $1^{o}$ aquecimento; (b) $2^{\circ}$ aquecimento; (c) $1^{\circ}$ arrefecimento. $m=$ $2,48 \mathrm{mg}$. Velocidade de aquecimento $10^{\circ} \mathrm{C} \mathrm{min}^{-1}$ (a)

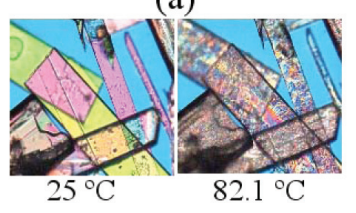

(b)

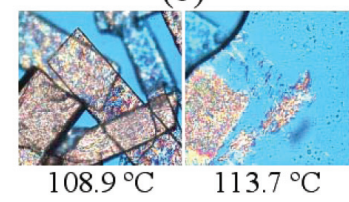

Figura 6. Imagens obtidas por termomicroscopia com luz polarizada de uma amostra de acetato de colesterol. (a) Ilustração da transição sólido/sólido; (b) ilustração da fusão. Velocidade de aquecimento $10^{\circ} \mathrm{C} \mathrm{min}^{-1}$. Ampliação $200 x$ (a)

$72.2^{\circ} \mathrm{C}$

$68.7^{\circ} \mathrm{C}$
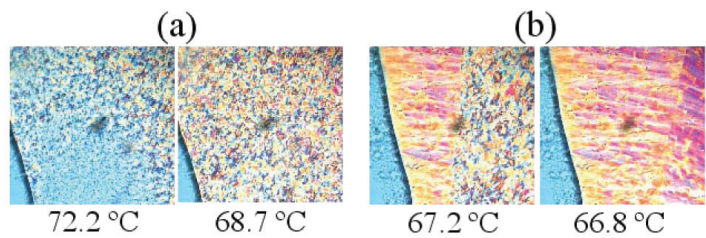

Figura 7. Imagens obtidas por termomicroscopia com luz polarizada durante o arrefecimento a $2,5^{\circ} \mathrm{C} /$ min de uma amostra de acetato de colesterol aquecida até $160^{\circ} \mathrm{C}$. (a) Formação da mesofase cristal líquido a partir do fundido; (b) transformação cristal líquido/sólido cristalino. Ampliação $200 x$

\section{CONCLUSÃO}

Neste trabalho é proposto um projecto laboratorial interdisciplinar que conjuga um método de síntese alternativo e conveniente, que utiliza um catalisador de fácil separação da mistura de reacção e que pode ser reutilizado repetidamente sem diminuição da actividade, com a caracterização espectroscópica e por métodos de análise térmica de reagentes e/ou produtos. Os estudos por análise térmica são utilizados também para introdução dos conceitos de polimorfismo e mesofases.

\section{PROCEDIMENTO EXPERIMENTAL}

\section{Esterificação do colesterol}

Colocar num balão de fundo redondo $(500 \mathrm{~mL})$, equipado com um condensador de refluxo, $10 \mathrm{~g}$ de argila Montmorillonite K10 e
$6 \mathrm{~mL}$ de anidrido acético dissolvido em $200 \mathrm{~mL}$ de diclorometano. Finalmente, adicionar colesterol (2,75 g, 0,007 mol). Manter a mistura em refluxo durante aproximadamente $5 \mathrm{~h}$.

Na aula seguinte, a argila K10 foi filtrada com papel de filtro num funil de vidro, e lavada com $\mathrm{CH}_{2} \mathrm{Cl}_{2}$. Uma amostra do filtrado foi analisada por cromatografia gasosa e mostrou que se obteve o acetato de colesterol com $95 \%$ de pureza. Foi utilizado como padrão o acetato de colesterol obtido comercialmente. O solvente é evaporado num evaporador rotativo. Rendimento de $80 \%$. O sólido obtido foi recristalizado segundo o procedimento usual: $\mathrm{O}$ acetato de colesterol $(100 \mathrm{mg})$ foi dissolvido em acetato de etilo: $\mathrm{CH}_{2} \mathrm{Cl}_{2}$ (10:1). A solução foi aquecida a $40{ }^{\circ} \mathrm{C}$ até se obter a solubilização completa. $\mathrm{O}$ balão foi colocado em repouso, no frigorífico, até à aula seguinte. Rendimento da recristalização $90 \%$.

\section{Recristalização do colesterol}

Colesterol $(200 \mathrm{mg})$ foram dissolvidos em $10 \mathrm{~mL}$ de metanol e 5 $\mathrm{mL}$ de diclorometano. Após dissolução por aquecimento à temperatura de refluxo o balão ficou em repouso até à aula seguinte. Procedeu-se a filtração e secagem dos cristais, tal como descrito anteriormente.

\section{Espectroscopia de ressonância magnética nuclear}

Os espectros de ressonância magnética nuclear ${ }^{1} \mathrm{H}$ e ${ }^{13} \mathrm{C}$ das soluções em $\mathrm{CDCl}_{3}$ foram obtidos em espectrómetros Bruker Avance 300 que opera a $300.13 \mathrm{MHz}$ para ${ }^{1} \mathrm{H}$ e a $75.47 \mathrm{MHz}$ para ${ }^{13} \mathrm{C}$, respectivamente. Os desvios químicos de ${ }^{1} \mathrm{H}$ estão expressos em $\delta$ (ppm) relativamente a um padrão de tetrametilsilano (TMS).

\section{Espectroscopia de infravermelho}

Os espectros de infravermelho dos compostos sólidos foram obtidos com um espectrofotómetro de infravermelho com transformada de Fourier ThermoNicolet IR 300 com resolução $1 \mathrm{~cm}^{-1}$. Para obtenção dos espectros recorreu-se à técnica de pastilha de brometo de potássio: aproximadamente $1 \mathrm{mg}$ do composto a estudar foi disperso em cerca de 100 $\mathrm{mg}$ de brometo de potássio e a mistura prensada a $10 \mathrm{t}$ durante $15 \mathrm{~min}$.

\section{Calorimetria diferencial de varrimento}

Nos estudos de calorimetria diferencial de varrimento foi utilizado o calorímetro Perkin-Elmer, modelo DSC7, calorímetro de compensação de potência, utilizando o Intracooler, com circulação de etilenoglicol/ $\mathrm{H}_{2} \mathrm{O}(1: 1 \mathrm{v} / \mathrm{v})$ a $-10{ }^{\circ} \mathrm{C}$, como sistema de arrefecimento. Utilizou-se como gás de purga azoto com elevada pureza e com fluxo de $20 \mathrm{~cm}^{3} / \mathrm{min}$.

A calibração de temperatura foi efectuada utilizando compostos referidos na literatura como materiais de referência: ${ }^{22}$ índio (material de referência, Perkin Elmer, 99,99\%; $\Delta_{\text {fus }} H=(3286 \pm 13) \mathrm{J} \mathrm{mol}^{-1}$; $T_{\text {fus }}=\left(156,60^{\circ} \mathrm{C}\right)$ ) e bifenilo (CRM LGC $2610, T_{\text {fus }}=68,93 \pm 0,03^{\circ} \mathrm{C}$ ). Para a calibração de entalpia utilizou-se como substância padrão o índio.

As amostras em estudo, com massas compreendidas entre 2 e 3 $\mathrm{mg}$, foram fechadas em cápsulas de alumínio de $25 \mu \mathrm{L}$ e submetidas a ciclos de aquecimento/ arrefecimento. Cada amostra foi sujeita a aquecimento até à fusão, seguindo-se o processo de arrefecimento até $25^{\circ} \mathrm{C}$. Terminado este processo efectuou-se um novo ciclo de aquecimento/ arrefecimento. Todos os varrimentos foram efectuados a $10{ }^{\circ} \mathrm{C} \mathrm{min}{ }^{-1}$.

\section{Termomicroscopia com luz polarizada}

As experiências foram realizadas com um sistema de aquecimento/arrefecimento Linkam DSC600 associado a um microscópio de transmissão de luz polarizada, DMRB da Leica. O microscópio é 
acoplado a uma câmara de vídeo Sony CCD-IRIS/RGB. As imagens foram obtidas combinando luz plano polarizada e compensadores de onda. Utilizou-se uma amplificação de 200x.

\section{MATERIAL SUPLEMENTAR}

No material suplementar, disponível gratuitamente em http:// quimicanova.sbq.org.br na forma de arquivo PDF, são apresentados o espectro de $\mathrm{RMN}$ de ${ }^{13} \mathrm{C}$ do acetato de colesterol em $\mathrm{CDCl}_{3}$, Figura $1 \mathrm{~S}$, e imagens obtidas por termomicroscopia ilustrativas dos dois tipos de comportamento observados para o acetato de colesterol no arrefecimento do fundido, Figura 2S.

\section{AGRADECIMENTOS}

Aos alunos de estágio laboratorial no Grupo de Catálise e Química Fina e à Fundação para a Ciência e a Tecnologia (FCT) pelo financiamento do projecto de investigação PTDC/QUI/66015/2006. R. M. D. Nunes agradece a bolsa SFRH/BD/24005/2005. MarvinSketch, ChemAxon (www.chemaxon.com), foi utilizado para desenhar as estruturas.

\section{REFERÊNCIAS}

1. Hochberg, R. B.; Endocrine Reviews 1998, 19, 331

2. Figueiredo, L. F.; Pereira, M. M.; Faria, J.; Catalysis from Theory to Application - An Integrated Course, Imprensa da Universidade de Coimbra: Coimbra, 2008.
3. Trost, B. M.; Science 1991, 254, 1471.

4. Garti, N.; Karpuj, L.; Sarig, S.; Thermochim. Acta 1980, 35, 343.

5. Epand, R. M.; Bach, D.; Borochov, N.; Wachtel, E.; Biophys. J. 2000, 78,866 .

6. Kaloustian, J.; Pauli, A. -M.; De La Porte, P. L.; Lafont, H.; Portugal, H.; J. Therm. Anal. Cal. 2003, 71, 342.

7. Kaloustian, J.; De La Porte, P. L.; El-Moselhy, T.; Lafont, H.; Portugal, H.; J. Therm. Anal. Cal. 2005, 82, 331.

8. Hilfiker, R., ed.; Polymorphism in the Pharmaceutical Industry, WileyVCH: Weinheim, 2006.

9. August, O.; Glaxo looses fight to protect Zantac from copycat drug: Glaxo Wellcome, The Times, 8/7/1996.

10. Shargel, L.; Wu-Pong, S.; Yu, A. B. C.; Applied Biopharmaceutics \& Pharmacokinetics, $5^{\text {th }}$ ed., Mcgraw-Hill: New York, 2005.

11. Barral, E. M.; Porter, R. S.; Johnson, J. F.; J. Phys. Chem. 1967, 71, 1224.

12. Price, F. P.; Wendorff, J. H.; J. Phys. Chem. 1971, 75, 2849.

13. Kunihisa, K. S.; Gotoh, M.; Mol. Cryst. Liq. Cryst. 1977, 42, 97.

14. Glagovich, N. M.; Shine, T. D.; J. Chem. Educ. 2005, 82, 1382.

15. Yadav, G. D.; Krisham, M. S.; Org. Process. Res. Devel. 1998, 2, 86.

16. Li, A.-X.; Li, T.-S.; Ding, T.-H.; Chem. Commun. 1997, 15, 1389.

17. Popjak, G.; Edmond, J.; Anet, F. A. L.; Easton Jr, N. R.; J. Am. Chem. Soc. 1977, 99, 931.

18. Wunderlich, B.; Thermochim. Acta 1990, 162, 59.

19. Gilson, D. F. R.; J. Chem. Educ. 1992, 69, 23.

20. Epand, R. M.; Bach, D.; Epand, R. F.; Borochov, N.; Wachtel, E.; Biophys. J. 2001, 81, 1511.

21. Reitnitzer, F.; Monatsh. Chem. 1888, 9, 421.

22. Sabbah, R.; Wu, A.X.; Chickos, J.S.; Leitão, M.L.P.; Roux, M.V.; Torres, L.A.; Thermochim. Acta 1999, 331. 93. 


\section{ARGILAS COMO CATALISADORES VERDES NA ESTERIFICAÇÃO DO COLESTEROL. CARACTERIZAÇÃO ESPECTROSCÓPICA E IDENTIFICAÇÃO DE POLIMORFOS POR MÉTODOS DE ANÁLISE TÉRMICA. UMA PROPOSTA LABORATORIAL INTERDISCIPLINAR PARA O $1^{\circ}$ CICLO UNIVERSITÁRIO}

Teresa M. R. Maria*, Rui M. D. Nunes, Mariette M. Pereira e M. Ermelinda S. Eusébio

Departamento de Química, Universidade de Coimbra, 3004-535 Coimbra - Portugal

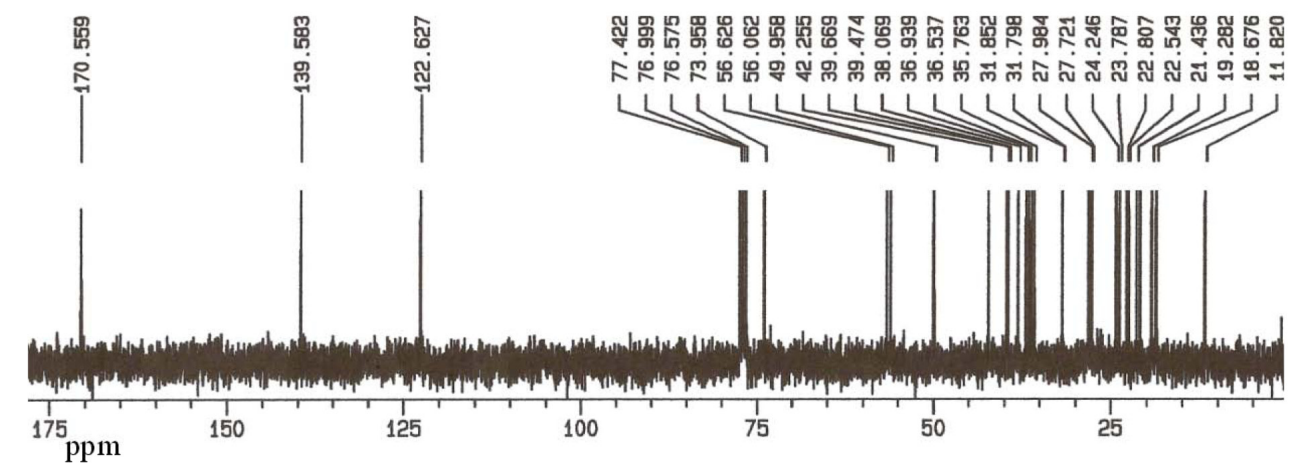

Figura 1S. Espectro de ${ }^{13} \mathrm{C} \mathrm{RMN}$ de acetato de colesterol em $\mathrm{CDCl}_{3}$

(a)

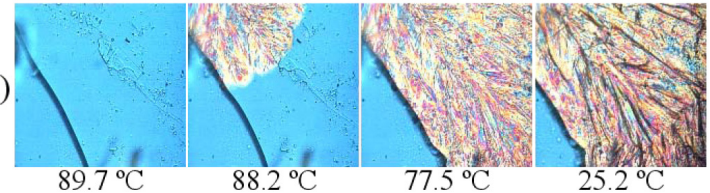

(b)

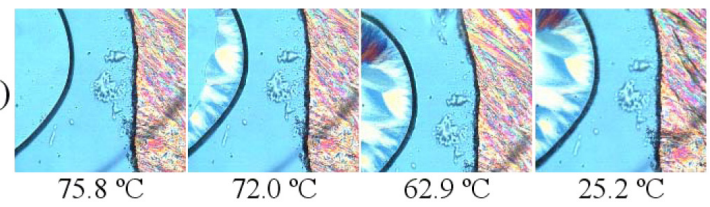

(c)

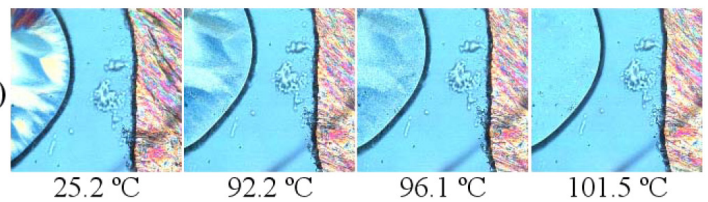

(d)

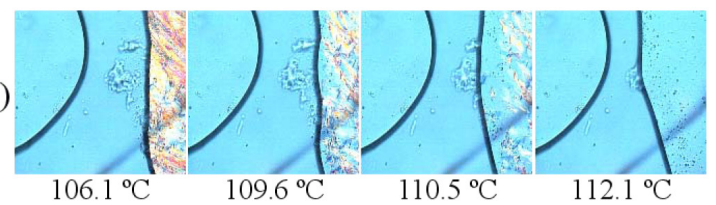

Figura 2S. Imagens obtidas por termomicroscopia com luz polarizada: (a) e (b) arrefecimento de amostras de acetato de colesterol a $2,5^{\circ} \mathrm{C} /$ min; $(c)$ e (d) aquecimento da amostra (b) a $10^{\circ} \mathrm{C} \mathrm{min}^{-1}$. Ampliação $200 x$ 